Günter Radden

\title{
Der verkannte $e$-Dativ im heutigen Deutsch: im Kreise meiner Lieben und am Rande des Abgrunds
}

\begin{abstract}
Der $e$-Dativ wurde bisher vornehmlich unter dem Aspekt seines fortschreitenden Rückgangs sowie seines antiquierten und erstarrten Status betrachtet. Tatsächlich ist der $e$-Dativ im gegenwärtigen Deutsch durchaus noch gebräuchlich. Bei den Substantiven Rand, Fall und Sinn ist der e-Dativ sogar beträchtlich häufiger belegt als der flexionslose Dativ. Diese Substantive bezeichnen unbelebte Referenten und kommen als Argumentdative somit nicht in Betracht. Der $e$-Dativ tritt vornehmlich als Adjunkt in Präpositionalphrasen auf, typischerweise mit einem Genitivattribut wie in am Rande der Verzweiflung. Diese Konstruktion mit ihren beiden möglichen Dativformen ist Gegenstand der Untersuchung. Die Wahl des $\emptyset$-Dativs oder $e$-Dativs ist vorwiegend durch die Semantik des Genitivattributs bestimmt. Die beiden Dativformen bilden die Endpunkte eines semantischen Kontinuums und lassen sich als Schema verstehen. Die Häufigkeitsverteilungen von $\emptyset$-Dativ und $e$-Dativ weisen darauf hin, dass der $e$-Dativ vor allem durch einen höheren Grad an menschlicher Involviertheit gekennzeichnet ist. Gegenüber dem Argumentdativ sowie dem endungslosen Adjunkt-Dativ nimmt der e-Dativ damit eine semantische Nische ein, der er bis heute sein „Fortleben“ verdankt.
\end{abstract}

\section{Einführung}

Dieses Kapitel befasst sich mit der Verwendung des $e$-Dativs gegenüber dem flexionslosen Dativ bei starken Maskulina und Neutra des Deutschen. Mehrere Arbeiten (u. a. Pfeffer \& Janda 1982, Rieger 2007, Konopka 2012) haben festgestellt, dass der Gebrauch des $e$-Dativs im gegenwärtigen Deutsch ,weitgehend verschwunden“ ist (Ebert 1993: 81) und nur noch in archaischem Stil und festen Verbindungen verwendet wird. Diese Generalisierung erweist sich bei genauerer Betrachtung jedoch als zu undifferenziert.

Wertvolle Hinweise und weiterführende Anregungen verdanke ich Thomas Berg, Klaus-Uwe Panther und zwei anonymen Gutachter/innen.

Günter Radden, Institut für Anglistik und Amerikanistik, Universität Hamburg 
Am Beispiel der beiden Substantive Kreis und Rand wird in dieser explorativen Studie gezeigt, dass der $e$-Dativ im heutigen Deutsch durchaus noch gebräuchlich ist. Die Untersuchung basiert auf Häufigkeitsverteilungen von im Kreis(e) und am Rand(e) mit einem folgenden Genitivattribut. Das Substantiv Kreis repräsentiert die Gruppe der starken Maskulina und Neutra, bei denen der $e$-Dativ bereits zurückgedrängt aber noch nicht verschwunden ist, während das Substantiv Rand die zweifellos kleinere Gruppe starker Maskulina und Neutra repräsentiert, bei denen der $e$-Dativ noch immer gebräuchlicher ist als der flexionslose Dativ, bzw. Ø-Dativ. Ziel der Untersuchung ist, die spezifische Verwendung und Bedeutung des $e$-Dativs gegenüber dem $\emptyset$-Dativ aufzuzeigen und seine Motivation nachzuvollziehen.

Ausgangspunkt der Untersuchung ist die Entwicklung des e-Dativs, die in Abschnitt 2 kurz an Verlaufskurven skizziert wird. Abschnitt 3 gibt einen kurzen Überblick zum Forschungsstand des $e$-Dativs und formuliert Fragestellungen für die Untersuchung. Abschnitt 4 argumentiert für eine synchrone Interpretation des $e$-Dativs im Rahmen eines kognitiv-linguistischen Ansatzes. Abschnitt 5 befasst sich mit den Häufigkeitsverteilungen von im Kreis gegenüber im Kreise, Abschnitt 6 mit den Häufigkeitsverteilungen von am Rand gegenüber am Rande. Die Untersuchungsergebnisse werden in Abschnitt 7 zusammengefasst und diskutiert.

\section{Entwicklung des $e$-Dativs}

Das sogenannte Dativ-e bezeichnet die dativische Flexionsendung auf $-e$ bei Maskulina und Neutra Singular der starken $a$-Deklination urgermanischen Ursprungs. Bereits im Mittelhochdeutschen wurde das dativische Flexiv $-e$ weitgehend apokopiert (für einen historischen und regionalen Überblick siehe Wegera 1987 und Ebert 1993) ${ }^{1}$. Der endungslose Dativ setzte sich zunächst in der gesprochenen Sprache durch und ist inzwischen auch in der Schriftsprache verbreitet.

In Wortverlaufskurven wird ersichtlich, dass der Anstieg des Ø-Dativs häufig mit einem gleichzeitigen Rückgang des $e$-Dativs verbunden ist. Die Referenz- und Zeitungskorpora des DWDS zeigen die Entwicklung der Häufigkeit von Wörtern von 1600 bis 2018 auf. Für die Thematik dieser Studie werden jedoch die Verläufe

\footnotetext{
1 Nach Ebert et al. (1993:118) hatte sich die -e-Apokope bereits im Zeitraum III (1450-1500) im gesamten oberdeutschen Raum durchgesetzt. Die regionalen Entwicklungen verliefen jedoch unterschiedlich: So fand im Schwäbischen bereits nahezu $100 \%$ Apokope statt, im Thüringischen jedoch kaum.
} 
ab dem 20. Jahrhundert entscheidend sein, d. h. ab dem Zeitraum, in dem sich der Rückgang des $e$-Dativs am deutlichsten manifestiert. Abbildung 1 gibt die Verlaufskurven der Sequenzen von im Kreis der und im Kreise der relativ zu einer Million Tokens wieder. Der Artikel der begrenzt die Suche auf Verbindungen der Substantive mit einem folgenden Genitivattribut. Er wird als Lemma interpretiert und umfasst daher auch den Artikel des.

\section{im @Kreis der · im @Kreise der - Verlaufskurve}

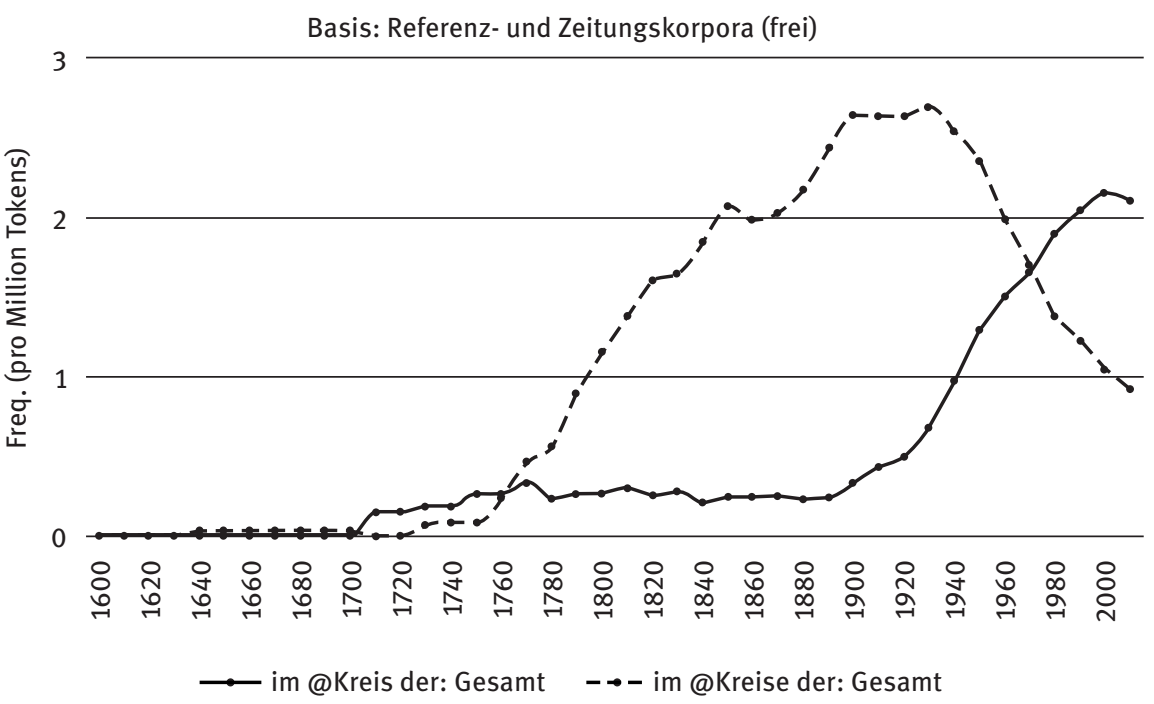

Abb. 1: Verlaufskurven von im Kreis der und im Kreise der von 1600 bis 2018. Durchgehende Linien stellen die Entwicklung des Ø-Dativs dar, gestrichelte Linien die des $e$-Dativs.

Die Frequenz von im Kreise in Abbildung 1 steigt ab Mitte des 18. Jahrhunderts kontinuierlich an und fällt nach einem Scheitelpunkt um 1940 abrupt zurück, während die Frequenz von im Kreis später ansteigt und deutlich die Verlaufskurve von im Kreise übersteigt. Vergleichbare Rückgänge des $e$-Dativs zu Beginn des 20. Jahrhunderts verbunden mit gleichzeitigem Anstieg des Ø-Dativs über den e-Dativ hinaus weisen u. a. auch die Substantive Jahr, Land, Fall, Tag und Weg auf. Diese Substantive bestätigen somit den generellen Rückgang des $e$-Dativs zu Gunsten des Ø-Dativs. Der Rückgang des $e$-Dativs im 20. Jahrhundert zeigt sich ebenfalls deutlich in den Verlaufskurven des Google Books Ngram Viewer für den Zeitraum von 1800 bis 2008.

Eine Verdrängung des $e$-Dativs durch den $\emptyset$-Dativ trifft jedoch nicht auf alle $e$-Dativ-fähigen Substantive zu. Der $e$-Dativ in der Präpositionalphrase am Rande ist nicht rückläufig, sondern im Gegenteil weiterhin ansteigend. 
Die Verlaufskurven in Abbildung 2 weisen einen nur schwachen Anstieg des $\emptyset$ Dativs Rand auf, aber einen rapiden Anstieg des $e$-Dativs Rande ab Beginn des 20. Jahrhunderts. Eine entsprechende rapide ansteigende Entwicklung weist auch die Sequenz im Zuge der auf. Mehrere weitere Substantive haben generell höhere Frequenzen des $e$-Dativs gegenüber dem $\emptyset$-Dativ, meist jedoch mit fallender Tendenz in neuerer Zeit. Dies trifft u. a. zu auf die Sequenzen im Sinne der, im Geiste der, im Lichte der und im Laufe der.

am @Rand der·am @Rande der - Verlaufskurve

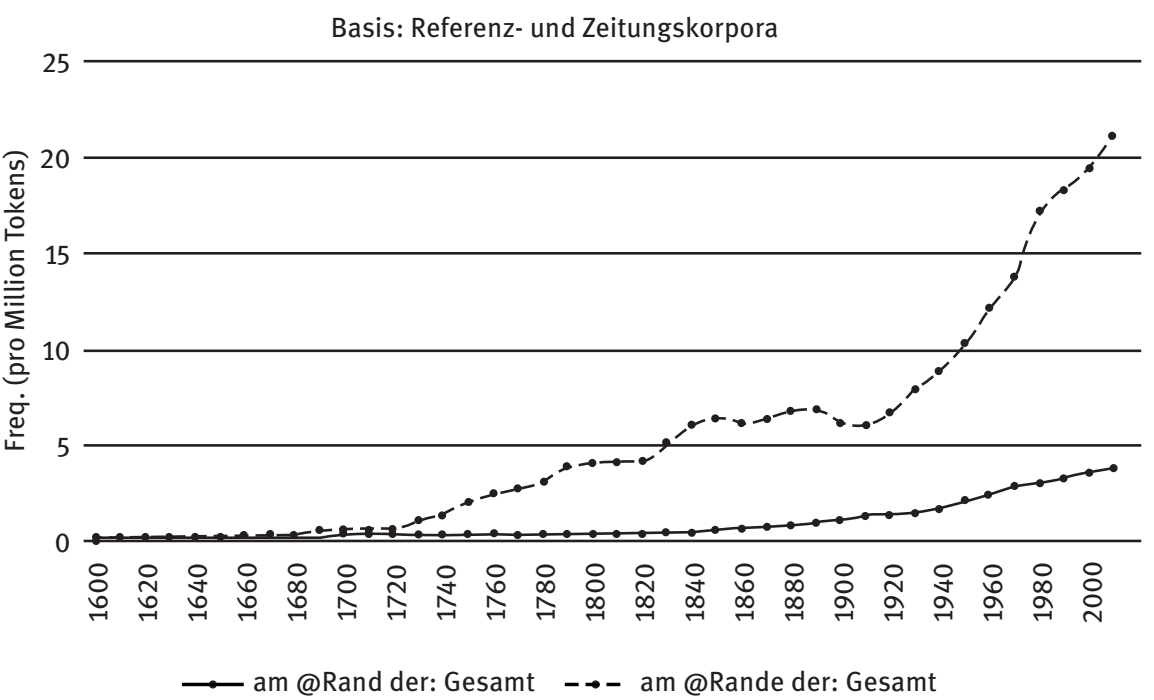

Abb. 2: Verlaufskurven von am Rand der und am Rande der von 1600 bis 2018. Durchgehende Linien stellen die Entwicklung des $\emptyset$-Dativs dar, gestrichelte Linien die des $e$-Dativs.

Die meisten Substantive, in denen beide konkurrierenden Dativformen gebräuchlich sind, zeichnen sich durch ihre figurative Bedeutung aus. So ist die Verwendung des Substantivs Lauf in der Phrase im Lauf(e) der Zeit metaphorisch motiviert durch die konzeptuelle Metapher ZEIT IST RAUM. Die beiden Dativformen unterscheiden sich jedoch hinsichtlich der Intensität und Komplexität der figurativen Bedeutung. Dies wird an den neueren Verwendungen von im Lauf(e) in Kollokation mit Genitivattributen im DWDS-Kernkorpus 21 (2000-2010) deutlich. So fällt von den 59 Treffern des Ø-Dativs von Lauf ein erheblicher Anteil auf die beiden temporalen Genitivattribute Zeit und Jahr, die, wie unter (1a) angeführt, jeweils $22 \%$ der Treffer ausmachen. Mit dem Ø-Dativ werden somit nur sehr generelle Zeitbegriffe kodiert. Der $e$-Dativ von Laufe wird ebenfalls mit temporalen 
Genitivattributen verwendet, die jedoch vielfältiger, spezifischer und komplexer sind. Unter (1b) sind einige modifizierte und spezifische Zeitangaben aufgeführt.

(1) a. im Lauf der Zeit (22\%), im Lauf der Jahre (22\%)

b. im Laufe der vergangenen Jahre, im Laufe der folgenden Nacht, im Laufe des Abends

c. im Laufe der Geschichte, im Laufe der Therapie, im Laufe des Studiums

Die unter (1c) aufgeführten Zeitangaben beziehen sich auf die Dauer von Ereignissen. Ereignisse stehen somit metonymisch für die Zeit der Ereignisse, und die Zeit wird metaphorisch als Raum verstanden. Die mit dem $e$-Dativ verbundenen Bedeutungen sind somit konzeptuell komplexer.

Der Sprachwandel führte offensichtlich zu einer funktionalen Aufspaltung der Kategorie Dativ mit dem Ergebnis, dass dem $\emptyset$-Dativ eine relativ generelle und unmarkierte Funktion und dem e-Dativ eine spezifische und markierte Funktion zukam bzw. gegenwärtig noch zukommt. Die vorliegende Studie beschränkt sich auf die synchrone Untersuchung der beiden Präpositionalphrasen im Kreis(e) und am Rand(e) mit ihren Genitivattributen, die sich deutlich hinsichtlich ihrer neueren Entwicklungen ab Beginn des 20. Jahrhunderts unterscheiden. Die Präpositionalphrase im Kreis(e) steht exemplarisch für den rückläufigen Trend des $e$-Dativs, dessen noch bestehende Verwendung jedoch Aufschlüsse über Faktoren gestattet, die möglicherweise für den Erhalt des $e$-Dativs verantwortlich sind. Die Präpositionalphrase am Rand(e) steht dagegen für die aktive Verwendung des $e$-Dativs, die sich in vergleichbarer Deutlichkeit bei keinem anderen Substantiv zeigt. Insbesondere das Beispiel von am Rand(e) zeigt deutlich, dass pauschale Erklärungsmuster für den spezifischen Gebrauch des $e$-Dativs nicht greifen, sondern präzisere Erklärungen erfordern.

\section{Bisherige Arbeiten zum Dativ-e bzw. e-Dativ}

Der $e$-Dativ wurde bisher vorwiegend hinsichtlich seiner Gebrauchsbedingungen sowie seines gegenwärtig erstarrten und antiquierten Status untersucht. Die semantischen und phonologischen Restriktionen des Dativ-e wurden bereits von Otto Behaghel (1909: 34) beschrieben. Er unterscheidet „Fälle, wo das e nicht gesetzt werden darf [. . . ] und Fälle, wo das e fehlen oder gesetzt werden kann.“ Die meisten Arbeiten zum $e$-Dativ befassen sich mit Behaghels zweiter Gruppe von Fällen, in denen das $e$ fehlen oder gesetzt werden kann, wie in $z u$ Haus(e) und im Kreis(e). 
Der Rückgang des $e$-Dativs wurde zuerst von Pfeffer und Janda (1982) statistisch erfasst: Der Anteil des $e$-Dativs an den Dativen belief sich in den 1960er Jahren auf lediglich 5\%. Der stärkste gegenwärtige Gebrauch des $e$-Dativs wurde von Konopka (2012) in festen idiomatischen Verbindungen festgestellt, wie in zu Grabe tragen (99,04\%), auf dem Weg nach Hause (98,75\%) und zu Grunde gehen (98,65\%). Die Verwendung erstarrter Phraseologismen wie zu Tode betrübt und im Grunde seines Herzens wurde von Rieger (2007) als stilistisch antiquiert, gehoben, feierlich, altehrwürdig und hochtrabend-pathetisch charakterisiert. Rieger weist auch auf die Bedeutung der Formkomponente hin. So ist die Metrik bedeutsam in zu Tode betrübt, Assonanz in sich etwas zu Gemüte führen, und Redeflusserleichterung durch Vermeidung einer Konsonantenfolge in zu Gebote stehen. Die Ergebnisse dieser Arbeiten schlagen sich auch in der impressionistischen Charakterisierung des Dativ-e im Duden, Richtiges und gutes Deutsch (2011: 224) nieder:

Die Endung -e im Dativ Singular starker Maskulina und Neutra ist nicht mehr erforderlich und wird nur selten gesetzt. In festen Redewendungen und formelhaften Verbindungen hat sie sich noch ziemlich fest gehalten: im Grunde genommen, zu Pferde sitzen, zu Kreuze kriechen, im Zuge sein, zu Buche schlagen u. Ä.

Interessanterweise führt auch der Duden ausschließlich Beispiele des $e$-Dativs in Präpositionalphrasen an. Hier liegt offensichtlich ein entscheidender, aber kaum berücksichtigter Schlüssel für das Fortleben des $e$-Dativs. In diesem Zusammenhang sind $\mathrm{u}$. a. die folgenden traditionellen Annahmen zum $e$-Dativ zu hinterfragen, auf die in Abschnitt 7 eingegangen wird:

(i) Ist der $e$-Dativ auf erstarrte Phraseologismen beschränkt?

(ii) Ist der $e$-Dativ eine fakultative Variante des $\emptyset$-Dativs?

(iii) Ist der $e$-Dativ mit einer eigenen Bedeutung verbunden?

\section{Synchrone Reinterpretation des $e$-Dativs}

Das Verhältnis der beiden Dativformen kann aus verschiedener Perspektive betrachtet werden. Die traditionelle diachrone Betrachtung legt das Augenmerk auf die Apokope des Dativ-e. Dabei wird implizit davon ausgegangen, dass der $\varnothing$-Dativ und der $e$-Dativ fakultative Varianten sind, denen dieselbe Funktion zukommt, nämlich den Kasus Dativ zu markieren. Tatsächlich ist eher davon auszugehen, dass den beiden Kasusformen in den Stadien ihrer Entwicklung verschiedene Funktionen zukamen. Zu Beginn der Aufspaltung des Dativs bildete der $e$-Dativ die unmarkierte Form und der neu gebildete Ø-Dativ die mar- 
kierte Form. In der weiteren Entwicklung wurde das Dativ-e wahrscheinlich als funktionslos empfunden, da die Funktion des Dativs bereits durch den Artikel, die Satzstruktur und die Verbsemantik erkennbar und somit das Gelingen der Kommunikation gewährleistet war (vgl. Moser 2001: 165).

Bei synchroner Betrachtung liegt das Augenmerk auf der Koexistenz und Konkurrenz der beiden Formen. Dabei sieht der gegenwärtige Sprachbenutzer im Dativ-e wohl kaum noch eine verblasste Kasusform, sondern eher die Epenthese eines e zum endungslosen Dativ. Aufgrund seiner größeren Vorkommenshäufigkeit bildet der $\emptyset$-Dativ inzwischen die unmarkierte, oder Default-Form des Dativs, und der $e$-Dativ die markierte Dativform. Der $e$-Dativ unterscheidet sich vom $\emptyset$-Dativ durch seine erweiterte Form:

(1) Das Dativ-e wird als Schwa hinzugefügt: im Kreis > im Kreise.

(2) Ein konsonantischer Auslaut wird damit zum vokalischen Auslaut.

(3) Eine Auslautverhärtung wird aufgehoben: /hont/ > /hondə/.

(4) Ein einsilbiges Substantiv wird zweisilbig, ein zweisilbiges dreisilbig, etc.

Der $e$-Dativ weist gegenüber dem Ø-Dativ in den Merkmalen (1) bis (4) ein Mehr an Form auf. Entsprechend dem ikonischen Prinzip der Isomorphie (Haiman 1980: 516) werden unterschiedliche Formen mit unterschiedlichen Bedeutungen verbunden, und nach dem ikonischen Prinzip der Quantität entspricht einer größeren morphologischen Komplexität eine größere semantische Komplexität. ${ }^{2}$ Wenn sich ein Sprecher für eine komplexere morphologische Form wie den $e$-Dativ entscheidet, könnte er damit beabsichtigen, seiner Aussage größere Intensität, Expressivität, Emotionalität oder Involviertheit zu verleihen. Dies könnte auch auf die antiquierten, altehrwürdigen und hochtrabend-pathetischen Bedeutungen zutreffen, die Rieger und Konopka in festen Phraseologismen mit dem $e$-Dativ feststellten.

Ziel der vorliegenden Untersuchung ist, die Faktoren zu bestimmen, die für die Präferenz des $\emptyset$-Dativs oder des $e$-Dativs verantwortlich sind. Dies lässt sich am besten in Präpositionalphrasen mit einem Genitivattribut aufzeigen, wie im Kreis(e) der Familie. Diese Konstruktion ist nicht nur mit beiden Dativformen häufig belegt, sondern enthält auch mit dem Genitivattribut eine Konstituente, die entscheidend zur Präferenz einer der Dativformen beiträgt.

In einer gebrauchsbasierten Beschreibung von $\emptyset$-Dativ und $e$-Dativ ist die Wahl zwischen den beiden Dativformen nicht kategorischer, sondern gradueller Natur. Der $\emptyset$-Dativ und der $e$-Dativ bilden die Endpunkte eines Kontinuums, das

2 Haiman (1980: 528): „[. . .] increased morphological complexity is an icon of increased semantic complexity.“ 
sich als Schema verstehen lässt im Sinne einer „ausdrucksseitigen Gestalt“, die „ein bestimmtes Konzept [...] mehr oder weniger zuverlässig repräsentiert“ (Köpcke 2000: 164). Die ausdrucksseitige Gestalt des Dativschemas ist durch die beiden Dativformen gegeben, und die Konzepte, die sie repräsentierten, spiegeln sich mehr oder weniger zuverlässig in ihren relativen Häufigkeiten wider. Zur Unterscheidung der als Adjunkte verwendeten Dativformen gegenüber dem Argumentdativ werden diese als Adjunkt-Dative und das Schema entsprechend als Adjunkt-Dativschema bezeichnet. Die beiden folgenden Abschnitte untersuchen die anteiligen Häufigkeiten beider Dativformen mit verschiedenen Genitivattributen. Abschnitt 5 befasst sich mit der Verwendung von im Kreis(e), Abschnitt 6 mit der Verwendung von am Rand(e).

Die Häufigkeiten der Dativ-Verwendungen von $\operatorname{im} \operatorname{Kreis}(e)$ und am Rand(e) wurden aus den Referenz- und Zeitungskorpora des DWDS ${ }^{3}$ ermittelt. Dieses große Metakorpus umfasst vorwiegend Daten aus dem 20. und frühen 21. Jahrhundert. Allerdings erweist sich das Korpus in manchen Fällen quantitativ als nicht repräsentativ genug und qualitativ zu stark auf Schriftsprache fixiert, so dass bestimmte Verwendungen nicht belegt sind. Um in diesen Fällen dennoch einen Eindruck von sprachlichen Ausdrucksmöglichkeiten zu gewinnen, wurden notfalls auch Daten aus Google herangezogen, ohne jedoch den angegebenen Häufigkeiten statistische Bedeutung zuzumessen. ${ }^{4}$

\section{Häufigkeitsverteilungen des Adjunkt- Dativschemas mit im Kreis(e)}

Die aus den Korpora des DWDS ermittelten Häufigkeiten von im Kreis und im Kreise (zuletzt abgerufen am 09.06.2020) sind in Tabelle 1 aufgeführt. Es sind jeweils die absoluten Häufigkeiten der beiden Dativformen sowie die auf den $e$-Dativ entfallenden abgerundeten prozentualen Anteile angegeben. Zeile (a) führt die Häufigkeiten von im Kreis(e) ohne Berücksichtigung der Umgebung auf, Zeile (b) die Häufigkeiten von $\operatorname{im} \operatorname{Kreis}(e)$ mit einem Artikel eines unbenannten Genitivattributs, und Zeilen (c) bis (e) geben die Häufigkeiten von im Kreis(e) mit einem benannten Genitivattribut.

3 Die Referenz- und Zeitungskorpora des DWDS umfassen sieben Korpora für den Zeitraum von 1473 (Deutsches Textarchiv) bis 2018 (DIE ZEIT) und sind frei verfügbar.

4 Auf die Problematik von Google-Trefferzahlen zur Häufigkeitsbestimmung haben Mann und Schierholz in einem Lexikographieblog sowie ein anonymer Gutachter dankenswerterweise hingewiesen. 
Tab. 1: Häufigkeitsverteilungen des Adjunkt-Dativschemas von im Kreis(e).

\begin{tabular}{llrrr}
\hline & & $\emptyset$-Dativ & $\boldsymbol{e}$-Dativ & Anteil $\boldsymbol{e}$-Dativ \\
\hline (a) & im Kreis $(e)$ & 9.043 & 5.142 & $36 \%$ \\
(b) & im Kreis(e) der & 2.119 & 1.460 & $41 \%$ \\
\hline (c) & im Kreis(e) der Freunde & 29 & 14 & $33 \%$ \\
(d) & im Kreis $(e)$ der Kollegen & 20 & 12 & $37 \%$ \\
(e) & im Kreis(e) der Familie & 166 & 154 & $48 \%$ \\
\hline
\end{tabular}

Zeile (a) zeigt, dass der e-Dativ mit einem Anteil von 36\% gegenüber dem $\emptyset$-Dativ bereits beträchtlich an Boden verloren hat. Der Anteil der $e$-Dative in Kollokation mit einem Genitivattribut erhöht sich in Zeile (b) auf $41 \%$. Lediglich die drei Genitivattribute unter (c), (d) und (e) konnten mit einer nennenswerten Frequenz aufgeführt werden. Einzig die Folge im Kreis(e) der Familie in Zeile (e) ist relativ häufig belegt und hat mit $48 \%$ einen hohen Anteil an $e$-Dativen. Dies legt nahe, dass die Semantik der Genitivattribute die Wahl der Dativform prägt und der Familie offenbar eine besondere Bedeutung zukommt.

Auch das Determinativum des Genitivattributs kann für die Wahl der Dativform bedeutsam sein. Außer dem definiten Artikel kommen insbesondere Possessiva und Demonstrativa in Betracht. Tabelle 2 kontrastiert die Sequenz im Kreis(e) der Familie mit der Sequenz im Kreis(e) seiner Familie.

Tab. 2: Häufigkeitsverteilungen des Adjunkt-Dativschemas von im Kreis(e) mit verschiedenen Determinativa.

\begin{tabular}{lcrrr}
\hline & & $\emptyset$-Dativ & e-Dativ & Anteil $\boldsymbol{e}$-Dativ \\
\hline (a) & im Kreis(e) der Familie & 166 & 154 & $48 \%$ \\
(b) & im Kreis(e) seiner Familie & 156 & 221 & $59 \%$ \\
\hline
\end{tabular}

Der Possessivum seiner in (b) erhöht deutlich die Frequenz des e-Dativs, wahrscheinlich, weil es der persönlichen Beziehung eines Referenten zu seiner Familie Ausdruck verleiht. Für eine separate Suche der einzelnen Determinativa fallen die meisten Häufigkeiten leider zu gering aus. Es wurde daher in Tabelle 3 die Abstandsangabe „\#2“ eingefügt, die zwei weitere Wörter zwischen Kreis(e) und dem genitivischen Substantiv zulässt. Dabei sind auch andere verbindende Wörter als Funktionswörter möglich, wie im Kreise guter Freunde. 
Tab. 3: Häufigkeitsverteilungen des Adjunkt-Dativschemas von im Kreis(e) und Genitivattributen.

\begin{tabular}{llrrr}
\hline & & $\boldsymbol{\emptyset}$-Dativ & $\boldsymbol{e}$-Dativ & Anteil $\boldsymbol{e}$-Dativ \\
\hline (a) & im Kreis(e) \#2 Freunde & 82 & 89 & $52 \%$ \\
(b) & im Kreis(e) \#2 Kollegen & 55 & 68 & $55 \%$ \\
(c) & im Kreis(e) \#2 Familie & 399 & 491 & $56 \%$ \\
(d) & im Kreis(e) \#2 Kinder & 10 & 20 & $67 \%$ \\
(e) & im Kreis(e) \#2 Lieben & 26 & 99 & $79 \%$ \\
\hline
\end{tabular}

Alle Werte für die anteiligen $e$-Dative in Tabelle 3 liegen über $50 \%$ und somit auch über denen von Tabelle 1. Auch die Unterschiede zwischen den Genitivattributen werden in dieser Aufstellung deutlicher. Ein Freundeskreis besteht aus frei gewählten und losen Mitgliedern; in Zeile (a) hat dieser Kreis den niedrigsten Anteil an $e$-Dativen. Ein Kreis von Kollegen ist gegeben und wird in Zeile (b) mit einem leicht höheren Anteil an $e$-Dativen kodiert. Die hohen Werte in Zeile (c) deuten an, dass die Familie den Prototyp eines Kreises von Personen darstellt. Die Familie bildet mit ihren Kindern den Kern jeder Gesellschaft. Die Nennung von Kindern in Zeile (d) erhöht erheblich den Anteil des $e$-Dativs. Einen noch höheren Anteil an $e$-Dativen erzielt in Zeile (e) im Kreise (meiner, Deiner, seiner, ihrer, der) Lieben. Eine weitere Steigerung wäre ein Ausdruck wie im Kreise Deiner Liebsten, der in den DWDS-Korpora jedoch nicht erfasst wird, in Google aber 50.900 Treffer und einen $e$-Dativ-Anteil von $87 \%$ erzielt (abgerufen am 06.06.2020).

Das Fehlen solcher Daten zeigt die Grenzen korpusbasierter Untersuchungen. Dies betrifft auch das Medium der Schriftsprache und seine Funktion der Berichterstattung. Es fehlt die mit dem ich verbundene Sprecherperspektive. So ist in Tabelle 2 das Possessivum seiner 221-mal mit dem $e$-Dativ belegt, die Possessiva meiner und unser jedoch nur 9-mal bzw. 3-mal. Trotz dieser Mängel von Korpora zeigen die Häufigkeitsverteilungen von im $\operatorname{Kreis}(e)$ deutliche Korrelationen des $e$-Dativs bzw. $\emptyset$-Dativs mit seiner semantischen Umgebung.

Die quantitativen Unterschiede innerhalb des Adjunkt-Dativschemas sind auch qualitativ relevant. Sie spiegeln die prototypische Struktur der figurativen Kategorie Kreis wider. Die geometrische Form des Kreises ist eine geeignete Metapher für das kulturelle Konzept der Gruppenzugehörigkeit. Die Metapher des Kreises suggeriert Aspekte wie Gleichheit und Zusammenhalt seiner Mitglieder sowie Geschlossenheit und Abgrenzung gegenüber Nichtmitgliedern. In der Antike wurde der Kreis sogar als Symbol der Vollkommenheit, der Einheit und des Göttlichen angesehen. Je stärker die Kohärenz der Mitglieder eines Kreises ist 
und je mehr sich der Sprecher/Schreiber mit dem Kreis und seinen Mitgliedern identifiziert, umso größer ist die Präferenz für den $e$-Dativ. Dies betrifft in besonderem Maße den Kreis einer Familie mit Kindern. Zusammenfassend lässt sich somit feststellen, dass der $e$-Dativ von im Kreise mit starken positiv-emotionalen Bedeutungen verbunden ist.

\section{Häufigkeitsverteilungen des Adjunkt- Dativschemas mit am Rand(e)}

Wie bei der Analyse von im Kreis(e) wird am Rand(e) auf der Basis der Daten der DWDS-Korpora untersucht. Tabelle 4 zeigt die Gesamthäufigkeiten von am Rand(e) in Zeile (a) unabhängig von der Umgebung und in Zeile (b) mit dem definiten Artikel für ein unbenanntes Genitivattribut.

Tab. 4: Häufigkeitsverteilungen des Adjunkt-Dativschemas von am Rand(e) ohne und mit definitem Artikel.

\begin{tabular}{llrrr}
\hline & & ø-Dativ & e-Dativ & Anteil $\boldsymbol{e}$-Dativ \\
\hline (a) & am Rand(e) & 7.059 & 37.195 & $84 \%$ \\
(b) & am Rand(e) der & 3.844 & 22.255 & $85 \%$ \\
\hline
\end{tabular}

Die anteilige Häufigkeit des $e$-Dativs in am Rande ist erheblich höher als die von im Kreise - der e-Dativ könnte sogar als die unmarkierte Dativform angesehen werden. Anders als bei im Kreis(e) sind die Anteile des e-Dativs an den Gesamthäufigkeiten von am Rande ohne folgenden Artikel in (a) und von am Rand(e) der in (b) annähernd gleich. Das Determinativum des Genitivattributs hat offenbar keinen wesentlichen Einfluss auf die Wahl des Dativs. Tatsächlich sind häufig kaum andere Determinativa als der definite Artikel mit am Rand(e) zu erwarten. Bildungen wie am Rande meines Existenzminimums oder am Rand eines Wahnsinns sind auch bei Google kaum belegt.

Die Häufigkeitsverteilungen von am Rand und am Rande variieren jedoch deutlich hinsichtlich der konzeptuellen Bereiche, die mit dem jeweiligen Genitivattribut verbunden sind. In Tabelle 5 werden fünf konzeptuelle Bereiche unterschieden, in denen am Rand (e) typischerweise auftritt: (a) Gegenstände, (b) Örtlichkeiten, (c) existentielle Notlagen, (d) psychische Ausnahmesituationen und (e) Zusammenkünfte. Wie bei den Suchanfragen von im Kreis(e) ist mit der Abstandsangabe „\#2“ die Möglichkeit für zwei weitere Wörter zwischen Rand(e) 
Tab. 5: Häufigkeitsverteilungen des Adjunkt-Dativschemas von am Rand(e) in verschiedenen konzeptuellen Bereichen.

\begin{tabular}{lllrrr}
\hline & Konzeptuelle Bereiche & & Ø-Dativ & e-Dativ & Anteil $\boldsymbol{e}$-D. \\
\hline (i) & Gegenstände & am Rand(e) \#2 Tisches & 7 & 4 & $36 \%$ \\
(ii) & Örtlichkeiten & am Rand(e) \#2 Autobahn & 28 & 53 & $65 \%$ \\
(iii) Existentielle Notlagen & am Rand(e) \#2 Abgrunds & 68 & 308 & $82 \%$ \\
(iv) Psychische Ausnahmesit. & am Rand(e) \#2 Wahnsinns & 6 & 74 & $92 \%$ \\
(v) Zusammenkünfte & am Rand(e) \#2 Veranstaltung & 6 & 155 & $95 \%$ \\
\hline
\end{tabular}

und dem Genitivattribut gegeben. Die fünf Bereiche sind entsprechend ihrem Anteil an $e$-Dativen angeordnet.

(i) Gegenstände: Ein Rand im konkreten Sinn bildet den äußeren Teilbereich eines Gegenstands. Für diese grundlegende Teil-Ganzes-Konstruktion finden sich im DWDS-Korpus erstaunlicherweise nur so wenig Treffer, dass sich ein systematischer Vergleich der beiden Dativformen erübrigt. So überwiegt in Zeile (i) der ØDativ in am Rand(e) des Tisches, während in am Rand(e) des Bildes der $e$-Dativ mit 6:10 Treffern überwiegen würde. Keine Belege enthält das Korpus für am Rand(e) der Torte, am Rand(e) des Handys, am Rand(e) des Auges, etc. Die entsprechenden Google-Ergebnisse weisen eine Präferenz für den $\emptyset$-Dativ auf, besonders deutlich in am Rand der Torte mit 68.300 Treffern gegenüber am Rande der Torte mit 24 Treffern (abgerufen am 09.06.2020).

(ii) Örtlichkeiten: Die Präpositionalphrase am Rand(e) der Straße beschreibt eine räumliche Relation zwischen der Örtlichkeit Straße und einem angrenzenden Randbereich. Der Randbereich kann innerhalb oder außerhalb der Örtlichkeit liegen, ist also nicht notwendig Teil des Ganzen. Die Auswahl der Beispiele in Tabelle 6 war bestimmt von einer Mindestgröße von 20 Vorkommen.

Tab. 6: Häufigkeitsverteilungen des Adjunkt-Dativschemas von am Rand(e) im Bereich ,Örtlichkeiten‘.

\begin{tabular}{llrrr}
\hline & Örtlichkeiten & Ø-Dativ & $\boldsymbol{e}$-Dativ & Anteil $\boldsymbol{e}$-Dativ \\
\hline (a) & am Rand(e) \#2 Straße & 76 & 81 & $52 \%$ \\
(b) & am Rand(e) \#2 Feldes & 26 & 44 & $63 \%$ \\
(c) & am Rand(e) \#2 Dorfes & 41 & 91 & $69 \%$ \\
(d) & am Rand(e) \#2 Parks & 21 & 78 & $79 \%$ \\
(e) & am Rand(e) \#2 Waldes & 23 & 104 & $82 \%$ \\
(f) & am Rand(e) \#2 Stadt & 132 & 694 & $84 \%$ \\
\hline
\end{tabular}


Der Anteil der $e$-Dative liegt bei allen Örtlichkeiten über dem der $\emptyset$-Dative, fällt jedoch besonders bei den ersten drei Örtlichkeiten (a) - (c) geringer aus als der Anteil von am Rande insgesamt (Tab. 4), d. h. die Verwendung des e-Dativs in Rande ist mit diesen Örtlichkeiten weniger typisch. Die Verwendung von Rand und Rande in Verbindung mit der Örtlichkeit Straße ist sogar annähernd ausgeglichen. Es lässt sich jedoch eine Präferenz für den Ø-Dativ erkennen, wenn der Rand der Straße als reine Ortsangabe fungiert, wie in „Sie steht am Rand der Straße“. Der $e$-Dativ wird dagegen bevorzugt, wenn am Rand der Straße ein Ereignis stattfindet. Beispiele aus dem DWDS-Korpus hierfür sind: „Kampiert haben sie am Rande der Straße“, „ein alter Mann, der verletzt am Rande der Straße liegt“, und „chinesische Militärlastwagen, die am Rande der Straße verrotten“. Ähnliches trifft auch auf die Örtlichkeiten unter Zeilen (b) und (c) zu. Personen „leben“ und Gebäude „stehen“ am Rand eines Dorfes, aber „Granaten schlugen am Rande des Dorfes ein“ und „die Hubschrauberbesatzung musste am Rande eines Dorfes zwischenlanden“. Der $e$-Dativ in am Rande ließe sich ikonisch als Hinweis auf die Intensität und Dauer eines auf dem Ort stattfindenden Ereignisses verstehen.

Die drei in den Zeilen (d) - (f) aufgeführten Örtlichkeiten sind deutlich stärker mit dem $e$-Dativ in Rande verbunden. Parks und Wälder sind mit positiven Einstellungen verbunden. Hotels, Villen und Paläste befinden sich am Rande von Parks, aber „Am Rand des Parks geschah jetzt die Katastrophe“. Die Beschreibung „Wir lebten in Connecticut am Rande eines Waldes“ wird zweifellos als sehr positiv empfunden, während die Aussage „Bäume am Rand des Waldes wachsen anders als jene, die mittendrin stehen“ eher als faktisch verstanden wird. Die deutliche Präferenz für den $e$-Dativ in am Rande der Stadt ist möglicherweise auf die Größe der Örtlichkeit und damit auch der Größe ihres Rands zurückzuführen.

(iii) Existentielle Notlagen: Drohende existentielle Notlagen werden metaphorisch als Rand eines Abgrunds konzeptualisiert. Die in Tabelle 7 aufgeführten Arten

Tab. 7: Häufigkeitsverteilungen des Adjunkt-Dativschemas von am Rand(e) im Bereich ,existentieller Notlagen'.

\begin{tabular}{llrrr}
\hline & Existentielle Notlagen & Ø-Dativ & e-Dativ & Anteil $e$-Dativ \\
\hline (a) & am Rand(e) \#2 Abgrunds & 68 & 308 & $82 \%$ \\
(b) & am Rand(e) \#2 Ruins & 23 & 97 & $83 \%$ \\
(c) & am Rand(e) \#2 Existenzminimums & 24 & 119 & $83 \%$ \\
(d) & am Rand(e) \#2 Zusammenbruchs & 10 & 91 & $90 \%$ \\
(e) & am Rand(e) \#2 Bankrotts & 7 & 107 & $94 \%$ \\
\hline
\end{tabular}


existentieller Notlagen werden deutlich mit dem $e$-Dativ in Rande ausgedrückt und bilden mit ihrem hohen Anteil an $e$-Dativen eine relativ homogene Gruppe.

In Zeile (a) ist das Abgrund-Szenario explizit benannt. Am Rande eines Abgrunds zu stehen ist für die meisten Menschen eine bedrohliche Vorstellung. Der Rand, auf dem man steht, ist zwar noch außerhalb des Abgrunds, aber wir haben die zwanghafte Vorstellung, in den Abgrund zu stürzen. Der Ort Rand steht somit metonymisch für ein Ereignis, das mit dem Ort assoziiert ist. Damit ist dieses beängstigende Szenario optimal geeignet als metaphorische Ausgangsdomäne für die Konzeptualisierung einer existentiellen Notlage als Zieldomäne. In den Korpusdaten wird der Ausdruck am Rand(e) des Abgrunds weniger für Personen verwendet, sondern fast ausschließlich figurativ für geographische Einheiten und Institutionen. Dabei stehen kleinere Einheiten wie Länder und Firmen eher am Rand des Abgrunds, größere Einheiten wie Kontinente, die Welt, die Wirtschaft und Parteien dagegen eher am Rande des Abgrunds.

Die Ausdrücke in Zeilen (b) bis (e) benennen die Art der existentiellen Notlage. Dabei evoziert zunächst am Rand(e) als Teil metonymisch den Abgrund als Ganzes, und die Metapher des Abgrund-Szenarios verleiht der existentiellen Notlage besondere Intensität. Mit Rand lassen sich auch positive Situationen verbinden, wie am Rande der Gesellschaft oder am Rande des Wohlstands. Interessanterweise evozieren diese positiven Vorstellungen ebenfalls bedrohliche „Abgründe“. ${ }^{5}$ Der Betrachter befindet sich gegenwärtig noch in einer positiven Lage, aber da er metaphorisch bereits am Rande eines Abgrunds steht, droht die Gefahr, dass er diesen Zustand verliert und in den Abgrund stürzt.

(iv) Psychische Ausnahmesituationen: Die bedrohende Vorstellung, in einen Abgrund zu stürzen, hat auch starke psychische Auswirkungen. Der Ausdruck am Rand(e) löst in Verbindung mit einer psychischen Ausnahmesituation wie Verzweiflung ebenfalls die Vorstellung eines Abgrund-Szenarios aus. Drei solcher mit am Rand(e) beschriebenen psychischen Ausnahmesituationen sind in Tabelle 8 aufgeführt.

Wie bei existentiellen Notlagen überwiegt bei psychischen Ausnahmesituationen deutlich der $e$-Dativ. Die Anteile des $e$-Dativs spiegeln offensichtlich graduelle Unterschiede an emotionaler Intensität wider. Danach wäre eine Verzweiflung weniger intensiv empfunden als der Wahnsinn, und Wahnsinn weniger intensiv als

5 Im Transkript eines Radio-Gottesdienstes lautet eine Stelle: „Für alle Menschen, die am Rande des Lebens leben, am Rande des Wohlstands, am Rand der Freiheit und Sicherheit“. https://books.google.de/books?id=6CLWDwAAQBAJ\&pg=PA272\&dq=\%22am+Rande+des+Wohl stands $\% 22 \& h \mathrm{l}=$ de\&sa $=$ X\&ved=0ahUKEwj16fqIj_PpAhVJCewKHRN7DpUQ6AEIMTAB\#v=onepa ge\&q=\%22am\%20Rande\%20des\%20Wohlstands\%22\&f=false (abgerufen am 08.06.2020). 
Tab. 8: Häufigkeitsverteilungen des Adjunkt-Dativschemas von am Rand(e) im Bereich ,psychischer Ausnahmesituationen‘.

\begin{tabular}{llrrr}
\hline & Psychische Ausnahmesituationen & $\boldsymbol{\emptyset}$-Dativ & $\boldsymbol{e}$-Dativ & Anteil $\boldsymbol{e}$-Dativ \\
\hline (a) & am Rand(e) \#2 Verzweiflung & 11 & 41 & $80 \%$ \\
(b) & am Rand(e) \#2 Wahnsinns & 6 & 75 & $93 \%$ \\
(c) & am Rand(e) \#2 Nervenzusammenbruchs & 7 & 193 & $97 \%$ \\
\hline
\end{tabular}

ein Nervenzusammenbruch. Sogar am Rande des Todes wird mit einem $e$-Dativanteil von $73 \%$ (mit 10 gegenüber 27 Treffern) offenbar als weniger dramatisch empfunden - möglicherweise, weil der Tod letztendlich ein natürliches Ereignis darstellt. Schwächere Ausdrücke negativer psychischer Zustände wie Enttäuschung, Frustration oder Sorge und positive Zustände wie Hoffnung, Besserung oder Erleichterung sind in Verbindung mit am Rande kaum oder gar nicht in den Korpora oder in Google belegt, was wiederum als Hinweis für die Rolle des $e$-Dativs zum Ausdruck intensiver negativer Ausnahmesituationen zu werten ist. Soweit Ausdrücke positiver Zustände mit am Rande vereinbar sind, drücken sie einen erhofften, aber kaum realisierbaren Übergang in den positiven Zustand aus. So bedeutet am Rande der Hoffnung - der Titel eines Reports über überfüllte griechische Flüchtlingscamps, ${ }^{6}$ dass sich Personen gegenwärtig in einem hoffnungslosen Zustand befinden und nur wenig Hoffnung für eine Änderung zum Guten besteht. Die Bedeutung von am Rande der Hoffnung steht somit diametral gegenüber der von am Rande des Wohlstands.

(v) Zusammenkünfte: Unter Zusammenkünften werden hier mehr oder weniger offizielle Veranstaltungen, Konferenzen, Treffen, etc. verstanden. Ein Ausdruck wie am Rand(e) der Konferenz weist auf Ereignisse außerhalb eines regulären Programms hin wie „Vieraugengespräche“. Diese „Randereignisse“ fallen zeitlich und örtlich mit der Konferenz zusammen, sind häufig aber bedeutsamer und aus Sicht der Medien häufig berichtenswerter als die offizielle Zusammenkunft selbst. Die Häufigkeiten der bekanntesten Arten von Zusammenkünften sind in Tabelle 9 aufgeführt.

Die Häufigkeitsverteilungen der Dative entsprechend der Arten von Zusammenkünften sind überraschend und gleichzeitig eindeutig. Alle Arten von $\mathrm{Zu}$ sammenkünften werden unterschiedslos und fast ausschließlich mit dem $e$-Dativ gebildet. Diese Ergebnisse decken sich allerdings nicht mit den Daten einer Google-Abfrage. Soweit die Google-Daten zuverlässig sind, entfallen auf am Rande

6 Sebastian Schneider, Am Rande der Hoffnung. http://www.amrandederhoffnung.tilda.ws. (abgerufen am 06.06.2020). 
Tab. 9: Häufigkeitsverteilungen des Adjunkt-Dativschemas von am Rand(e) im Bereich ,Zusammenkünfte‘.

\begin{tabular}{llrrr}
\hline & Zusammenkünfte & $\emptyset$-Dativ & e-Dativ & Anteil $\boldsymbol{e}$-Dativ \\
\hline (a) & am Rand(e) \#2 Veranstaltung & 6 & 266 & $98 \%$ \\
(b) & am Rand(e) \#2 Parteitags & 3 & 119 & $98 \%$ \\
(c) & am Rand(e) \#2 Konferenz & 8 & 331 & $98 \%$ \\
(d) & am Rand(e) \#2 Sitzung & 3 & 249 & $99 \%$ \\
(e) & am Rand(e) \#2 Treffens & 3 & 653 & $100 \%$ \\
\hline
\end{tabular}

des Parteitags $94 \%$ und auf am Rande des Treffens $83 \%$ der Dative. Bei allen anderen Arten von Zusammenkünften liegt der $e$-Dativ sogar unter dem der $\emptyset$-Dative (abgerufen am 09.06.2020). Diese Differenzen erklären sich wahrscheinlich aus der Basis der DWDS-Daten, die überwiegend auf Zeitungen beruhen, vornehmlich DIE ZEIT, Tagesspiegel und Berliner Zeitung. Somit könnten die hohen Werte des $e$-Dativs durch die Thematik der meist politischen Berichterstattungen motiviert gewesen sein. Die Thematik solcher Treffen ist gesellschaftlich relevant, und Gespräche am Rande eines Treffens ganz besonders.

Interessanterweise weisen auch private Treffen hohe $e$-Dativ-Werte auf, insbesondere, wenn ihnen ein besonderer Anlass zu Grunde liegt. Hierzu gibt es leider keine hinreichenden Daten in den DWDS-Korpora. In Google weist der $e$ Dativ in am Rand(e) der Feier einen Anteil von $85 \%$ auf und in am Rand(e) der Geburtstagsfeier sowie am Rande der Weihnachtsfeier sogar einen Anteil von $100 \%{ }^{7}$ Hinsichtlich der hohen Frequenz ihrer $e$-Dative sind so verschiedene Zusammenkünfte wie politische Treffen und Geburtstagsfeiern somit durchaus vergleichbar: Beide werden als besondere, relevante Ereignisse eingeschätzt.

Die Ergebnisse der Häufigkeitsverteilungen des Adjunkt-Dativschemas von am Rand(e) mit einem Genitivattribut lassen sich abschließend wie folgt zusammenfassen.

- Der Ø-Dativ in am Rand wird vorwiegend verwendet in Relationen zwischen einem Gegenstand und seinem Rand als Teil des Gegenstands (am Rand des Tisches).

- Der $e$-Dativ in am Rande wird vorwiegend verwendet (ii) bei einem mit dem Rand einer Örtlichkeit verbundenen Ereignis (am Rande der Straße kampieren), (iii) bei der bedrohlichen Vorstellung einer existentiellen Notlage ( $\mathrm{am}$

7 am Rand(e) der Feier: Ø-Dativ=17.900; e-Dativ=98.000; am Rand(e) der Geburtstagsfeier: $\emptyset$-Dativ = 5; $e$-Dativ = 4.710; am Rande der Weihnachtsfeier $\emptyset$-Dativ = 8; $e$-Dativ $=16.500$ (abgerufen am 09.06.2020). 
Rande des Ruins), (iv) bei der bedrohlichen Vorstellung einer psychischen Ausnahmesituation (am Rande des Wahnsinns) und (v) bei bedeutsamen offiziellen oder persönlichen Zusammenkünften (am Rande der Tagung).

- Die mit dem $e$-Dativ verbundenen Bedeutungsaspekte umfassen u. a. eine Verbindung mit Ereignissen ${ }^{8}$ und positiven Einstellungen (bei Örtlichkeiten), starke Intensität und Emotionalität (bei existentiellen Notlagen und psychischen Ausnahmesituationen) sowie Relevanz von Ereignissen (bei Zusammenkünften). In allen diesen Verwendungen des $e$-Dativs wird am Rande metaphorisch verwendet.

\section{Diskussion der Ergebnisse}

Die vorangehenden Analysen befassten sich mit den Häufigkeitsverteilungen von im Kreis(e) und am Rand(e) mit einem Genitivattribut. Der Schwerpunkt lag dabei auf der Verwendung des $e$-Dativs. Die in Abschnitt 3 formulierten drei Fragestellungen zum $e$-Dativ lassen sich wie folgt beantworten.

\section{(i) Ist der $e$-Dativ auf erstarrte Phraseologismen beschränkt?}

Die häufig genannte Annahme, dass der $e$-Dativ gegenwärtig nur noch in erstarrten Phraseologismen vorkommt, trifft zumindest für die hier untersuchten Substantive Kreis und Rand nicht zu. Beide Substantive sind noch relativ frei verwendbar und kompatibel mit attributiven Substantiven aus den verschiedensten konzeptuellen Bereichen. So kann die mit Kreise bezeichnete Gruppe von Mitgliedern spontan auf weitere Gruppen ausgedehnt werden, wie in Beispiel (2a) mit Bezug auf ein Politikertreffen. Hier ist die Wahl des $e$-Dativs offensichtlich motiviert durch die Bedeutung des Treffens, des begrenzten Teilnehmerkreises, des hohen Rangs der Teilnehmer sowie ihrer gemeinsamen Zielsetzung.

(2) a. Merkel im Kreise der Mächtigen. (DIE ZEIT 06.07.2017)

b. Nein, aus dem Kreise der Lieben wird niemand ausgestoßen. (Der Tagesspiegel 14.10.2004)

8 Der Bedeutung von Ereignissen für die Wahl des $e$-Dativs wird besonders deutlich in dem Kontrast zwischen der Ortsangabe in am Rande des Spielfelds mit einem Anteil von 67 \% (7 ØDative gegenüber $14 e$-Dativen) und dem Ereignis in am Rande des Spiels mit einem Anteil von $98 \%$ (2 Ø-Dative gegenüber $117 e$-Dativen). 
Die Wahl der Präposition ist dagegen weniger frei. So enthält das DWDS-Korpus zwar mehrere Belege für aus dem Kreise wie in Beispiel (2b), aber nicht, oder kaum, für über dem Kreise, nach dem Kreise, neben dem Kreise, etc.

\section{(ii) Ist der $e$-Dativ eine fakultative Variante des $\emptyset$-Dativs?}

Den Verwendungen der beiden Dativformen wurde bisher keine Aufmerksamkeit geschenkt, möglichweise unter der Annahme, dass es sich um fakultative Varianten des Dativs handelt. Dieselbe Situation kann durchaus mit dem $e$-Dativ oder dem Ø-Dativ ohne erkennbaren Bedeutungsunterschied beschrieben werden. So beschreiben die beiden Dativformen in den Schildern Warnung vor dem Hund und Warnung vor dem Hunde zweifellos denotativ denselben Sachverhalt und können als fakultative Varianten angesehen werden. Es muss jedoch auch der Tatsache Rechnung getragen werden, dass die beiden Dativformen teilweise beträchtliche Unterschiede hinsichtlich ihrer Frequenz und damit auch ihrer Verwendungen aufweisen. Die obigen Analysen haben gezeigt, dass in bestimmten konzeptuellen Bereichen Präferenzen für die eine oder andere Form bestehen. So ist selbst die deutliche Dominanz des e-Dativs in am Rande des Treffens mit 262 Treffern im DWDS gegenüber dem $\emptyset$-Dativ mit 2 Treffern präferenziell und nicht absolut. Die entscheidende Frage ist, welche Konzepte bzw. Bedeutungen die Dativformen „mehr oder weniger zuverlässig“ repräsentieren.

\section{(iii) Ist der $\boldsymbol{e}$-Dativ mit einer eigenen Bedeutung verbunden?}

Diese Frage wurde in der Literatur bisher nicht thematisiert. Wie mehrfach verdeutlicht wurde, sind mit dem $e$-Dativ verschiedene konnotative und implizierte Bedeutungsaspekte verbunden. Die volle Bedeutung, die eine Präpositionalkonstruktion mit dem $e$-Dativ repräsentiert, ergibt sich aus dem Zusammenwirken seiner Konstituenten. So vermittelt im Ausdruck im Kreise der Familie das Substantiv Familie einen hohen Grad an gesellschaftlich positiver Wertschätzung, das metaphorisch verwendete Substantiv Kreis ebenfalls einen hohen Grad an positiver Bindung, der $e$-Dativ eine Intensivierung dieser positiven menschlichen Aspekte, und ein Possessivum bringt darüber hinaus eine persönliche Beziehung zum Ausdruck, wie sie z. B. bei der Schilderung einer Weihnachtsfeier als „im Kreise seiner Familie“ angebracht wäre. In der Formulierung mit dem Ø-Dativ und dem Artikel, d. h. im Kreis der Familie, würden diese intensivierenden, positiven Bedeutungsaspekte fehlen. Analog steuert in am Rande der Verzweiflung das Substantiv 
Verzweiflung einen hohen Grad an Emotionalität bei, das metaphorisch verwendete Substantiv Rand die bevorstehende Bedrohung, und der $e$-Dativ eine Intensivierung der menschlichen Beteiligung in diesem Szenario, die wiederum in am Rand der Verzweiflung fehlen würde.

Die mit dem $e$-Dativ verbundenen implizierten Bedeutungen umfassen anthropozentrische Bereiche wie Emotionen, Empfindungen, mentale Einstellungen sowie Handlungen, die hier zusammenfassend als menschliche Involviertheit bezeichnet werden. Der Begriff der Involviertheit wird in der linguistischen Literatur unterschiedlich verstanden: als Involviertheit der Sprecherin / des Sprechers, als semantische Makrorolle und als ein semantisches Merkmal von Kasus. Die mögliche Relevanz dieser Verständnisse von Involviertheit für den $e$-Dativ soll kurz thematisiert werden.

Involviertheit wird von Chafe (1982) und Biber (1988) als ein Aspekt sprachlicher Variation verstanden. Auf Grund des on-line Charakters, der Informalität und der face-to-face-Interaktion weist die gesprochene Sprache ein hohes Maß an Involviertheit der Sprecherin / des Sprechers auf gegenüber der geschriebenen Sprache, die durch räumliche, zeitliche und personelle Distanz (detachment) gekennzeichnet ist. Sprecherinvolviertheit manifestiert sich in mehreren sprachlichen Bereichen, u. a. in „mentalen und psychologischen Verben“ (private verbs) wie think und feel, mit denen persönliche Einstellungen, Gedanken und Emotionen kommuniziert werden. Die Parallelität zu den Bedeutungsaspekten des $e$-Dativs ist unverkennbar. Ein Merkmal der Sprecherinvolviertheit in der vorliegenden Studie wäre der Gebrauch des Possessivums mein, für dessen Gebrauch die Frequenzen leider nicht ausreichten.

Im rollensemantischen Ansatz von Lehmann, Shin \& Verhoven (2004) und Primus (2006) bildet Involviertheit (Indirectus) eine von drei semantischen Makrorollen einer Situation. Auf einer Skala von kausaler Dependenz zwischen einer kontrollierenden Rolle (Actor) und einer affizierten Rolle (Undergoer) nimmt die Rolle Involviertheit eine mittlere Stellung ein. Involviertheit kann entweder mit Kontrolle bzw. Affiziertheit verbunden sein oder unabhängig von kausaler Dependenz sein. ${ }^{9}$ Der eigenständigen Makrorolle Involviertheit sind vor allem die Mikrorollen Experiens, Benefiziär, Emittent und Rezipient sowie lokale Rollen zugeordnet (Lehmann, Shin \& Verhoven 2004: 6). Die ersteren vier Mikrorollen der Involviertheit sind mit Belebtheit verbunden und werden im Deutschen typischerweise als Dativargument kodiert.

9 Ähnlich argumentiert Langacker (1991a: 327 und 1991b: 209-225) mit seinen Konzepten von role archetypes und canonical action: So erzeugt der aktive Partizipant (agent) Energie, die evtl. unter Beteiligung eines Instruments zum passiven Patiens übertragen und dort „absorbiert“ wird. 
Wegener (1985: 321) betrachtet „das Merkmal des Betroffenseins oder SichBetroffenfühlens [. . .] (BETR) als semantische Grundfunktion des Dativs“. Die semantischen Merkmale von BETR sind Belebtheit, Involviertheit und Agentivität. Belebtheit ist ein inhärentes Merkmal des Argumentdativs. Sätze mit einem nichtbelebten Dativ wie ${ }^{\star}$ Er baut dem Auto eine Garage sind daher ungrammatisch. ${ }^{10}$ Das Belebtheitsmerkmal besagt für die Dativ-NP: „das in ihr genannte belebte Wesen ist nicht nur als objektiv von der Handlung Betroffener zu sehen, sondern als potentiell sich betroffen Fühlender, der an der Handlung als Beobachter, Empfindender oder gar Handlungsteilnehmender konkret beteiligt sein kann“ (Wegener 1985: 286). Involviertheit ordnet Wegener nach dem Akkusativ und dem Nominativ erst an letzter Stelle dem Dativ zu. Sie beschränkt Involviertheit dabei „auf passives Involviertsein (Handlungsunterworfensein)“ (Wegener 1985: 294). Agentivität ist nach dem Nominativ und vor dem Akkusativ mit dem Dativ verbunden. Involviertheit und Agentivität sind in diesem Modell somit zwar bedeutsame Merkmale des Dativs, ihm aber nicht inhärent. Die Beschränkung der Merkmale auf Beteiligung an Handlungen ist allerdings problematisch. So weist Langacker (1991a: 327 und 1991b: 221-222) darauf hin, dass der Experiencer u. a. sogar als Possessor kognitiv aktiv ist in dem Sinne, dass er mentale Kontakte und interne Repräsentationen initiiert.

Das Merkmal der Belebtheit des dativischen Referenten ist unstrittig. Die weiteren Merkmale des Dativs sind schwer abgrenzbar und werden hier unter dem Merkmal der menschlichen Involviertheit zusammengefasst. Involviertheit charakterisiert die Sprache der Sprecherin / des Sprechers, ist eine Makrorolle und subsumiert verschiedene Mikrorollen, insbesondere Experiens, zeichnet sich durch passives sowie auch aktives Involviertsein aus, und enthält als Merkmal der Belebtheit die menschlichen Aspekte des „Betroffenseins“ oder „Sich-Betroffenfühlens“.

Die mit dem Argumentdativ verbundenen Merkmale sind durchaus vergleichbar mit den Merkmalen, die den e-Dativ von im Kreise und am Rande sowie wohl auch anderer Substantive in Präpositionaladjunkten charakterisieren. Der einzige, aber erhebliche Unterschied zwischen dem Argumentdativ und dem $e$-Dativ besteht darin, dass der Argumentdativ belebte Wesen und ihre menschliche Involviertheit bezeichnet, während der $e$-Dativ nichtbelebte Entitäten bezeichnet, gleichzeitig aber menschliche Involviertheit impliziert.

10 Wegener (1985: 286) kommentiert ungrammatikalische Beispiele dieser Art anschaulich: "Für die Tatsache, daß die Kasusform des Dativs mit Belebtheit assoziiert ist, spricht allein schon die Tatsache, daß ungrammatische Sätze wie die obigen bei den Informanten Belustigung und Kommentare wie ,Dann muß man sein Auto aber sehr lieben . . . ' hervorrufen [. . . ]“ 
Der Dativ als Argument wird fast nur noch flexionslos und nicht (mehr) mit dem Dativ-e gebildet, da dessen Belebtheitsbedeutungen bereits im Dativ enthalten sind. Dem $e$-Dativ verbleibt somit nur die grammatische Funktion eines Adjunkts in Form einer Präpositionalphrase. Wie in Abbildung 3 dargestellt, nimmt der adjunktive $e$-Dativ eine Zwischenstellung zwischen dem Argumentdativ und dem adjunktiven $\emptyset$-Dativ ein. Er teilt mit dem $\emptyset$-Dativ das Vorkommen in einer Präpositionalphrase sowie das referentielle Merkmal der Unbelebtheit. Der $e$-Dativ und der $\emptyset$-Dativ unterscheiden sich als Endpunkte des Adjunkt-Dativschemas nur graduell hinsichtlich des implizierten Grads an menschlicher Involviertheit. Der $e$-Dativ weist einen hohen Grad an Involviertheit auf, der $\varnothing$-Dativ keine oder geringere Involviertheit. Das Merkmal der menschlichen Involviertheit hat der $e$-Dativ offensichtlich vom Argumentdativ übernommen, wobei „erstarrte Phraseologismen“ möglichweise bei der Übernahme von Aspekten der Involviertheit beigetragen haben.

\begin{tabular}{|c|c|c|c|c|}
\hline & \multirow[t]{2}{*}{ Argumentdativ } & \multicolumn{3}{|c|}{ Adjunktdative } \\
\hline \multirow{4}{*}{$\begin{array}{l}\text { Referent } \\
\text { Bedeutung }\end{array}$} & & $e$-Dativ & & $\emptyset$-Dativ \\
\hline & belebt & unbelebt & $=$ & unbelebt \\
\hline & & implizierte & & implizierte \\
\hline & Involviertheit $\longrightarrow$ & Involviertheit & $\mathrm{HH}$ & „Uninvolviertheit“ \\
\hline
\end{tabular}

Abb. 3: Argumentdativ und Adjunktdative. Der Pfeil steht für die Übertragung von Merkmalen, das Gleichheitszeichen für gemeinsame Merkmale, und die Skala für skalare Merkmale.

Unter den drei Dativformen stellt der $e$-Adjunktdativ tatsächlich nur noch ein peripheres Mitglied dar. In seiner formalen und semantischen Nähe zum $\varnothing$-Adjunktdativ und der implizierten inhaltlichen Nähe zum Argumentdativ nimmt der $e$-Dativ eine Zwitterstellung ein. Dank seiner eigenen distinktiven Form und seiner expressiven Bedeutung besetzt der $e$-Dativ jedoch eine ökologische Nische, der er bis heute sein „Fortleben“ verdankt. 


\section{Literatur}

Behaghel, Otto (1909): Der Dativ der Einzahl männlicher und sächlicher Hauptwörter.

Zeitschrift des allgemeinen Deutschen Sprachvereins 24/2, 33-39.

Biber, Douglas (1988): Variation across speech and writing. Cambridge: Cambridge University Press.

Chafe, Wallace L. (1982): Integration and involvement in speaking, writing and oral literature. In Deborah Tannen (Hrsg.), Spoken and written language: Exploring orality and literacy, 35-53. Norwood, NJ: Ablex.

Duden (2011): Richtiges und gutes Deutsch. 7. Aufl. Bd. 9. Mannheim: Duden Verlag.

Ebert, Robert Peter, Oskar Reichmann, Hans-Joachim Solms \& Klaus-Peter Wegera (1993): Frühneuhochdeutsche Grammatik. Tübingen: Niemeyer.

Haiman, John (1980): The iconicity of grammar: Isomorphism and motivation. Language $56,515-540$.

Köpcke, Klaus-Michael (2000): Starkes, Schwaches und Gemischtes in der Substantivflexion des Deutschen. Was weiß der Sprecher über Deklinationsparadigmen? In Rolf Thieroff, Matthias Tamrat, Nanna Fuhrhop \& Oliver Teuber (Hrsg.), Deutsche Grammatik in Theorie und Praxis, 155-170. Tübingen: Niemeyer.

Konopka, Marek (2012): Dem Manne kann geholfen werden - Wann kommt das Dativ-e zum Einsatz? In Marek Konopka \& Roman Schneider (Hrsg.), Grammatische Stolpersteine digital, 115-123. Mannheim: Institut für Deutsche Sprache.

Langacker, Ronald W. (1991a): Foundations of cognitive grammar, Volume II: Descriptive Application. Stanford: Stanford University Press.

Langacker, Ronald W. (1991b): Concept, image, and symbol: The cognitive basis of grammar. Berlin, New York: De Gruyter Mouton.

Lehmann, Christian, Yong-Min Shin \& Elisabeth Verhoeven (2004): Direkte und indirekte Partizipation: Zur Typologie der sprachlichen Repräsentation konzeptueller Relationen. 2. Aufl. Arbeitspapiere des Seminars für Sprachwissenschaft der Universität Erfurt 13.

Mann, Michael \& Stefan J. Schierholz: google-trefferzahlen zur häufigkeitsbestimmung? Lexikographieblog. https://lexikographieblog.wordpress.com/2014/10/07/google-treffer zahlen-zur-haufigkeitsbestimmung/ (abgerufen am 29.05.2020)

Moser, Hugo (2001): Beobachtungen zum heutigen Kasussystem und Kasusgebrauch. In Harald Scholler \& John Reidy (Hrsg.), Lexicography and dialect geography, 165-186. Wiesbaden: Franz Steiner.

Pfeffer, J. Alan \& Richard D. Janda (1982): Die Bildung des Dativs mit und ohne -e. In Jay Alan Pfeffer (Hrsg), Probleme der deskriptiven deutschen Grammatik, 34-46. Heidelberg: Julius Groos Verlag.

Primus, Beatrice (2006): Mismatches in semantic-role hierarchies and their dimensions of role semantics. In Ina Bornkessel, Matthias Schlesewsky, Bernard Comrie \& Angela D. Friedrici (Hrsg), Semantic role universals and argument linking: Theoretical, typological and psycholinguistic perspectives, 53-87. Berlin, New York: De Gruyter Mouton. 
Rieger, Marianne Christina (2007): Ein Dativ-e, wie es im Buche steht: Empirische Untersuchung der phraseologisch gebundenen Verwendung des Dativ-e. https://silo.tips/download/ ein-dativ-e-wie-es-im-buche-steht (abgerufen am 29. 05.2020)

Wegener, Heide (1985): Der Dativ im heutigen Deutsch. Tübingen: Gunter Narr Verlag. Wegera, Klaus-Peter (1987): Grammatik des Frühneuhochdeutschen: Beiträge zur Laut- und Formenlehre, Dritter Band: Flexion der Substantive. Heidelberg: Carl Winter. 
